\title{
RESEARCH METHODS IN LINGUISTICS: AN OVERVIEW
}

\section{Snejana Obeyd}

Department of English Studies, Konstantin Preslavsky University of Shumen, Bulgaria

\begin{abstract}
The paper offers a short theoretical overview of the main research paradigms, key issues, procedures, data collection and methods of analysis applied to the study of language. General description of qualitative, quantitative and mixedmethod research is followed by more detailed account of the three approaches. Different criteria for evaluating research are enumerated and specified and the most useful tools for obtaining quantitative data in linguistics are pointed out: questionnaire surveys and the experimental studies. The reviewed qualitative procedures leading to the generation of a set of data are ethnography, one-to-one interviews, focus group interviews, introspection, case studies, diary studies and research journals. Qualitative content analysis is a deeper level of analysis and interpretation of the underlying meaning of the data. Grounded theory has grown into the "mainstream" and many of its theoretical aspects are considered. The most significant of the discourse-analytic approaches: Conversation analysis, Discourse analysis, Critical discourse analysis and Feminist Post-Structuralist Discourse analysis are highlighted. The application of the discussed research paradigms to various research contexts is exemplified by reference to authors whose work is regularly published in SILC.
\end{abstract}

Key words: research, methods, analysis, qualitative, quantitative

\begin{abstract}
About the author: Senior Lecturer Snejana Obeyd is a PhD candidate working at the Department of English Studies at Konstantin Preslavsky University of Shumen, Bulgaria. She teaches courses in general and applied English to BA students in English Studies and Applied Linguistics with English and Russian. Her courses focus on translation from Bulgarian into English and vice versa. Her area of interest and research is text linguistics and translations.
\end{abstract}

e-mail: s.obeyd@shu.bg

ORCID iD: https://orcid.org/0000-0002-0676-5560

\section{Copyright (C) 2021 Snejana Obeyd}

Article history: Received: 1 February 2021; Reviewed: 20 February 2021; Revised: 25 March 2021; Accepted: 28 March 2021; Published: 19 April 2021

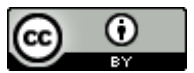

This open access article is published and distributed under a Creative Commons Attribution 4.0 International License.

Citation (APA): Obeyd, S. (2021). Research methods in linguistics: An overview. Studies in Linguistics, Culture, and FLT, 9(1), 54-82. doi: 10.46687/SILC.2021.v09i01.004 


\section{Introduction}

Research is done in order to provide new insights into a particular subject, to generate knowledge and thus assist in comprehending an issue. In scientific sense, research is "the organized, systematic search for answers to the questions we ask" (Hatch \& Lazaraton, 1991, p. 1). It is important for the researcher to become familiar with the principles of best practice. Different methods have been developed to collect and analyze data resulting in two research paradigms: qualitative and quantitative research. However, Brown (2001) argues that a more constructive approach is to view the two of them as "a matter of degrees" than "a clear-cut dichotomy". Hence, they are not mutually exclusive and a third branch "mixed methods research" is introduced, combining the data collection and the analysis levels of both qualitative and quantitative methods to best effect. The paper aims at providing a brief account of the three research methodology frameworks, their general characteristics and implications. It gives an overview of the key issues, questions, criteria and principles concerning research in linguistics. Different phases of quantitative data collection and analysis, and qualitative procedures are examined, focusing on description of discourseanalytic approaches to oral and written texts.

\section{Qualitative, quantitative, and mixed methods research}

Research involves collecting data, analyzing it and making inferences based on the analysis. Therefore, the key issue is data. In line with the basic research paradigms, there are three main types of data as outlined by Richards $(2005, \mathrm{p}$. 19):

- Quantitative data is expressed in numbers (for example, the number of times a linguistic item occurs in a specific context, resulting in numerical data analyzed by statistical methods);

- Qualitative data usually involves recorded oral data, transcribed to written form as well as written (field) notes and various kinds of documents, resulting in open-ended, non-numerical data further analyzed by non-statistical methods;

- Language data involves language samples, obtained from respondents for the purpose of language analysis. This type is often part of qualitative data.

As regards the numerical vs. non-numerical contrast between the two methods, which is too straightforward, Richards (2005) concludes "qualitative and quantitative data do not inhabit different worlds. They are different ways of recording observations of the same world" (pp. 3-6). In the same context, Miles and Huberman (1994) state that all data are qualitative because they refer to "essences of people, objects and situations" (p. 9). The main distinction lies in the nature of categories and the relevant process of the two methods. Categories 
or codes are used for structuring the information obtained. Bazeley (2003, p. 414) asserts that "Codes - the way they are generated, what they stand for, and the way they are used - lie at the heart of differences between quantitative and qualitative data and analysis tools". They also differ in their approach to the individual diversity of the participants examined and the results obtained. Quantitative researchers support a "meaning in the general" strategy, whereas qualitative researchers focus on a detailed understanding of the "meaning in the particular". Therefore, quantitative research could avoid individual respondent variation and the researcher's subjective interpretation which makes it more attractive in terms of its systematic nature managed by precise regulations. Whereas qualitative methods allow revealing subtle meanings and individual nuances and the attraction lies in the sensitivity to the individual. Quantitative research offers a structured macro-perspective of the general trends, while Qualitative research represents a context-sensitive micro-perspective of the world (Dörnyei, 2007, p. 27) If applied in appropriate contexts, the two approaches are equally valuable - a view referred to as the "situationalist" approach to research methodology. (Rossman \& Wilson, 1985). Hence, particular research issues are looked at from different angles, either from a macro-perspective or a microperspective thus unfolding different aspects of the issue in question. It is this mixing method that has great potential in research contexts.

\section{Quantitative research}

The progress of the natural sciences in the nineteenth century gave rise to quantitative social research and early researchers accepted and followed the "scientific method" in their studies. Generally, that method suggests three key stages in the research process: 1) observing a phenomenon; 2) creating an initial hypothesis; 3) testing the hypothesis by collecting and analyzing empirical data using systematic procedures. After the hypothesis has been tested and validated by recurrence, it is accepted as a scientific theory. Thus, the scientific method provided the tool to examine questions objectively. Francis Galton was the first to establish quantitative data collection and analytical methods in psychology at the turn of the twentieth century. That was the period dominated by quantitative methodology in the social sciences. This dominance started to change in the 1970s when research methodology started a process of reorganization. Nowadays, quantitative and qualitative methods coexist in different fields of social sciences.

The quantitative research is characterized with the use of numbers and they are enlivened to meaning when they are contextually backed up, i.e. we define the category the number stands for as well as the values within the variable. Another important feature is the theoretical categorization, i.e. the specification of categories is done before the study. Quantitative research is mainly focused on the common features of groups of people/things, operating with the concepts 
of averages and is less interested in individual cases. It deals with the study of variables expressing the common features. The variables are measured, thus, specifying the relationship between them. The most prominent quantifying feature is statistical analysis. The use of standardized measures eliminates subjectivity from the various phases of the research process. The specific aspect of quantitative research is going beyond the particular in the pursuit for generalizing the facts. In short, quantitative research has strengths that are indisputable - it is systematic, controlled, involving exact measurements resulting in reliable and generalized results. On the other hand, the method is not sensitive in unfolding the dynamics of the examined phenomenon. Reasonably enough it is considered as "overly simplistic, decontextualized, reductionist in terms of its generalizations, and failing to capture the meanings that actors attach to their lives and circumstances" (Brannen, 2005, p. 7).

\section{Qualitative research}

Qualitative research is really difficult to give an exact definition for it lacks a clear-cut set of methods and has "no theory or paradigm that is distinctly its own..." (Denzin \& Lincoln, 2005, p. 7). And then they concluded "Qualitative research is many things to many people" (p. 10). However, there are some peculiar features that need to be mentioned. The foremost feature of qualitative research is its emergent nature, i.e. no aspect of the research design is predicted, the study is flexible and open to new details that may emerge during the process. The investigation starts without hypotheses determined beforehand but gradually the focus of research becomes more salient and the analytic concepts are formed throughout the process of research. Another distinctive feature is the nature of qualitative data. That kind of research works with abundance of data (field notes, journal, diary entries, interviews, videos) transformed into a textual form. The common aim of all types of qualitative methods is to decode a set of meanings in the phenomena in question, so it is necessary for the data to include rich and complex details. Consequently, if a thorough description is aimed at achieving almost any appropriate information can be accepted as qualitative data. The research setting takes place in its natural environment without attempts to manipulate the situation. The research refers to the subjective opinions, experiences, feelings of individuals and thus the obvious goal of research is to explore the participants' views of the situation being studied. Meaning is a fundamental qualitative principle - only the participants themselves reveal the meanings and interpretations of their experiences. Hence the "insider perspective" is vital for researchers, i.e. they try to view and interpret a situation from the inside. Another characteristic feature is the small sample of participants involved due to the intensive and time-consuming research. Qualitative research analysis is basically interpretive, i.e. the final conclusions are the result of a researcher's subjective interpretation of the data. 
As Miles and Huberman (1994, p. 7) point out, "The researcher is essentially the main 'measurement device' in the study".

Qualitative research is effective in exploring new areas by studying and explaining in details a phenomenon. It does not rely on previous knowledge and empirical findings. The methods are used for decoding highly complex matters. The participant sensitivity of the research is a major factor in deciding the main points of the phenomenon requiring special attention as it gives preparatory guidelines validated by the respondents. Thus, grounding the research on such evidence we avoid intellectual manufacture focusing on the truth of the matter. The study has a flexible, emergent nature allowing for the researcher to look for answers to questions that have unexpectedly arisen. Duff (2008) emphasizes that 'instead of seeking a generalizable 'correct interpretation' qualitative research aims to broaden the repertoire of possible interpretations of human experience" (qtd. in Dörnyei, 2007, p. 39). The analysis is founded on rich data, thus, avoiding speculations as well as ensuring plenty of material for the report. An interesting example illustrating the method is the textual analysis of Ivanova (2020, pp. 16-35).

\section{Mixed methods research}

In the last 30 years, mixed method research has been considered a third approach in the methodology of research. It is a combination of research tools and techniques for the study of the same phenomenon. Miles and Huberman (1994, p. 310) best described the new emerging trend "Quantitative and qualitative inquiry can support and inform each other. Narratives and variable-driven analyses need to interpenetrate and inform each other. Realists, idealists, and critical theorists can do better by incorporating other ideas than by remaining pure. Think of it as hybrid vigour." In 1970s the term "triangulation" was introduced in relation to combining qualitative and quantitative data sources. Denzin (1978) supported triangulation as a way of validating hypotheses by exploring them by means of multiple methods. The mixed method has a lot of support and Lazaraton's statement is demonstrative in this respect "I would also hope that we would see more studies that combine qualitative and quantitative research methods, since each highlights 'reality' in a different, yet complementary, way" (2005, p. 207).

Mixed methods research can be specified as a combination of quantitative and qualitative methods in a single project. The real issue in the mixed-method paradigm concerns how the quantitative-qualitative combination takes place and for what purpose. In fact, different elements of the two paradigms can be combined in several ways:

- two types of research questions (with qualitative and quantitative approaches); 
- the manner in which the research questions are developed (participatory vs. preplanned);

- two types of sampling procedures (e.g., probability and purposive);

- two types of data collection procedures (e.g., focus groups and surveys);

- two types of data (e.g., numerical and textual);

- two types of data analysis (statistical and thematic), and

- two types of conclusions (representations, "objective" and "subjective", etc.). (Tashakkori \& Creswell, 2007, p. 4, qtd. in Litosseliti, 2010, p. 33).

Mixed method research is seen as "increasing the strengths" of one method while "eliminating the weaknesses" of another, which highlights the best of both paradigms. A quantitative phase can be followed by a qualitative element to balance the generalization issue by adding depth to analysis or results (Dörnyei, 2007, p.45). This method is appropriate for multi-level analysis of a complex phenomenon. We can obtain a clearer picture by linking up numbers and words. It also provides evidence for the research validity of the results, increasing their generalizability. That method more easily reaches a larger audience, offering something to everybody. Certain phenomena are best described using either qualitative or quantitative methods but the mixed method paradigm can present extra benefits for the comprehension of a phenomenon and it has the potential to contribute to high quality of research findings.

\section{Quality criteria}

\section{Quality criteria in quantitative research}

In doing quantitative research the reliability and validity of the methods and instruments of measurement have to be considered. Reliability refers to "consistencies of data, scores or observations obtained using elicitation instruments, which can include a range of tools from standardized tests administered in educational settings to tasks completed by participants in a research study" (Chalhoub-Deville, 2006, p. 2). Reliability is denoted by Rasinger as "measure repeatedly delivering the same (or near same) results" (Litosseliti, 2010, p. 55). There are different ways of checking reliability: testretest method measures the consistency of results when you do the same test again to the same sample at a different time; interrater is when the test is carried out by different people.: parallel forms - different versions of a test designed to measure the same thing: and internal consistency measures the consistency of the individual components of the test (Middleton, 2019). The other criterion for quality in quantitative research is validity, which term is simply explained by 
Dörnyei "a test is valid if it measures what it is supposed to measure" (2007, p. 51). Currently, the term points to the truthfulness of the interpretation of the test scores. According to Lynch (2003, p. 149) "validity is a property of the conclusions, interpretations or inferences that we draw from the assessment instruments and procedures, not the instruments and procedures themselves" (qtd. in Dörnyei, 2007, p. 52). Thus, content- and criterion-related evidence contribute to the overall validity construct together with validity analysis of the consequences of score interpretations and use. Bachman (2004) summarizes measurement validity in several main points: - validity is a quality of the interpretations; - perfect validity cannot be proven - only evidence could be provided that the argument is plausible enough; -validity is specific to every situation; -it can be supported with various kinds of evidence. Research validity is broader than measurement validity as it is related to the overall quality of the whole research project and more particularly to 1) the meaningfulness of the interpretations that researchers make on the basis of their observations, and 2) the extent to which these interpretations generalize beyond the research study (Bachman, 2004). Validity is divided into two types: internal and external. A study has internal validity, if the product is a function of the variables measured. External validity is the extent to which we generalize our findings to other contexts, i.e. when the result does not apply only to the original sample studied.

\section{Quality criteria in qualitative research}

Setting quality standards in qualitative research is problematic because the study is subjective, discursive and context-specific., i.e. "truth" is conditional and "facts" subjectivized (Morse \& Richards, 2002). There are three basic quality factors to be taken into account in qualitative research: - Insipid data - the quality of the analysis depends on the quality of the original data. If the data is not interesting, then we will obtain stereotypical results and "close to common sense", as Seale et. al. point out (Dörnyei, 2007, p. 55).; - Another important factor is the quality of the researcher, who is the "control centre", "the instrument", responsible for the whole procedure.; - Anecdotalism and the lack of quality safeguards - Usually researchers are limited in their presenting examples of the data that has led them to conclusions. Silverman, Miles and Huberman consider that the whole process and report could be put to doubt for there are not any essential safeguards against a biased conclusion (Dörnyei, 2007, p. 56).

The specific quality criteria are connected with reliability. It refers to the "degree of consistency with which instances are assigned to the same category by different observers or by the same observer on different occasions" (Silverman, 2005, p. 224). Kirk and Miller define reliability in field work as the extent to which the findings are not dependent on accidental factors. While Morse and 
Richard clarify the tendency of neglecting reliability in the past "reliability requires that the same results would be obtained if the study were replicated" (Dörnyei, 2007, p. 57). However, repetition of the process is problematic since the conclusions are a result of respondents' personal accounts and a researcher's subjective interpretation.

\section{Lincoln and Guba's taxonomy of quality criteria}

Lincoln and Guba (1985) introduced the term "trustworthiness" in relation to validity. They suggest four components that "trustworthiness" is composed of: - Credibility, or the "truth value" of the study, the qualitative equivalent of "internal validity"; -Transferability, or the applicability" of the results to other contexts, the qualitative parallel of "external validity"; - Dependability, or the "consistency" of the findings, the qualitative equivalent of "reliability"; - Confirmability, or the neutrality of the results, the qualitative counterpart of "objectivity" (Dörnyei, 2007, p. 57).

The following taxonomy presents various aspects of the traditional concepts of validity and reliability by Maxwell (1992 as qtd. in Dörnyei, 2007).

\section{Maxwell's taxonomy of validity in qualitative research}

Maxwell viewed the qualitative and quantitative approaches to validity as equally compatible. He proposed five components of validity:

- Descriptive validity refers to the factual precision of the researcher's report and is seen as the principal aspect whereas all the other categories of validity depend on it. It signifies the researcher's experience as well as the collection and interpretation of data based on other investigations, a strategy known as "investigator triangulation".

- Interpretative validity. Bearing in mind that descriptiveness underlies all validity aspects good research focuses on the interpretation of the real events, behaviours and objects by participants. The quality of the portrayal from the participants' perspective can be guaranteed by discussing the findings with them, i.e. to obtain feedback.

- Theoretical validity refers to the internal validity of the study, i.e. whether the researcher's report includes an appropriate theoretical basis and how well the theory characterizes the phenomenon in question.

- Generalizability. Maxwell (1992) described internal and external generalizability which concern the extension of the description to people, time and setting different from those studied directly. "Internal generalizability" is generalizing within the group observed while "external" one refers to generalizing to other groups. Generalizability in qualitative research has a different function from the one in the quantitative method and the "internal" 
one is more important for researchers. The main ideas generated through the process of theoretical expansion which is, in turn, eliqtd. from particular people or situations are applicable to other situations. Thus, specificity might lead to generalization. A strategy to test generalizability, offered by Duff (2006), is to take into consideration the participants' estimation about the generalizability of the issue in question.

- Evaluative validity refers to the researcher's evaluation of the targeted issue with regard to usefulness and practicability, i.e. how accurately the research analysis gives value judgement to the phenomenon. That aspect refers to the explicit and implicit use of an evaluation system in a qualitative analysis, investigating how the evaluative assertions fit the issue observed (Dörnyei, 2007, p. 59).

\section{Research design-based strategies}

These strategies provide the most convincing proof about research validity and they are an inherent part of the project.

-Method and data triangulation - the term "triangulation" refers to the use of multiple methods, sources or ideas in a research project. By exploiting triangulation, the possibility of biased conclusions in qualitative studies is efficiently reduced because the diverse data collection provides strong evidence. Yet, there is a question left unanswered: What will be the interpretation of any disagreement between the results?

- Prolonged engagement and persistent observation - engaging for a long time with the issue contributes to validity (Dörnyei, 2007, p. 62).

- Longitudinal research design - Duff (2006) states that longitudinal studies can open up various progressive ways and document different kinds of interaction.

\section{Quality criteria in mixed methods research}

Dörnyei (2007) suggests some aspects of the quality of mixed methods research to be considered:

- The rationale for mixing methods - It is worth mentioning that a mixed methods study offers a more in-depth means of making inferences acceptable. It combines the validity evidence of the qualitative and quantitative components, thus ensuring research validity;

- The "design validity" of the study. - The notion of "design validity" (Teddlie \& Tashakkori, 2003) concerns a new aspect of internal validity peculiar to mixed methods research. It is associated with the extent to which quantitative and qualitative components of a mixed methods research are combined so that the 
general design exhibits strengths and, weaknesses of the constituent methods, that would not overlap (Brewer \& Hunter, 1989; Tashakkori \& Teddlie, 1998). To support their assertions of a good design validity, researchers have to present evidence for two quality aspects: first, they have to prove the choice of the particular methods, integrated into the study, to be reasonable. The main principle should be centered around the "fitness-for-purpose" selection (Dörnyei, 2007, p. 63).

\section{Research questions and hypotheses}

The logical way to do research includes generating the research questions, choosing the design, then the method and the instruments allowing for the questions to be answered. However, the "research-question-first" principle does not always work when we know little of the topic.

Every project starts with a research topic which initially is usually a rather vague idea about the phenomenon. Then we need to state the purpose of the research, describing the objectives of the planned investigation focusing on the reason for launching the study and its potential importance. The next step towards "narrowing down" and "operationalizing" the research purpose is to define research questions. They are intended to convert the purpose into particular questions that the study is supposed to answer. Formulating the right questions is the basis of the research methodology for achieving the purpose. Johnson and Christensen (2004) argue that the research question is frequently omitted from the final report for it is predominantly a restatement of the purpose (Dörnyei, 2007 , p. 73). The good research questions have to postulate an interesting issue. As Gall et al. (2007, p. 41) put it "The imagination and insight that goes into defining the research problem usually determines the ultimate value of a research study more than any other factor" (Dörnyei, 2007, p. 73). The logical way to do research includes generating the research questions, choosing the design, then the method and the instruments allowing for the questions to be answered. Realising the possible outcomes of the investigation we can narrow down the scope of the research purpose to "research hypotheses" in place of, or in addition to, questions. Hypotheses are specific predictions about the results and the empirical study will either confirm or deny them. Nunan (1992) recommends how an investigation can be put to work. He suggests creating a "research outline" consisting of a plan with headings, each of which would involve a short statement. The headings have to refer to the research sphere, purpose, questions/hypotheses, the data collection method, type of data analysis (Dörnyei, 2007, p. 74). 


\section{Paradigmatic differences in formulating the research questions}

Qualitative and quantitative investigation processes are different in the specification of the purpose of study and consequently, in the questions to be explored. A well-specified quantitative purpose leads to formulating the target variables which, in turn, define concrete methodology. Research hypotheses are conceived on the basis of a researcher's predictions. An inherent feature of qualitative studies is their evolving nature and that is why qualitative research purposes and questions are often vaguer than their quantitative equivalents. The qualitative research purpose only specifies the central idea of the study with the intention of gaining new insights and probably theorizing about the issue explored. Accordingly, qualitative research questions are more general and explanatory, focusing on the "big picture" of the phenomenon.

Mixed methods studies characterized with their contradictory nature, require the definition of a purpose and questions that are both specific and vague. As Dörnyei (p. 74) proposes, a good strategy is to start with a general purpose statement, followed by a hypothesis for the specific mixed design and the methods to be applied, and concluded by research objectives and questions for the different research components. Creswell (2003) offers a practical model in mixed method research that consists of placing the questions at the beginning of each phase in the study.

Sunderland (2010) explores different types pf research questions with a focus on linguistic studies: - Primary/ secondary questions, depending on the focus of the study; - Main/ contributory; - Overarching/ subordinate questions, hierarchically ordered; - Empirical/ methodological/ theoretical, meant for working empirical findings; - Researcher-generated/ participant-generated; Empirical/ speculative questions (qtd. in Litosseliti, 2010, p. 16). The questions have to be related so that they could form a coherent unit. Good research questions are basic to a good research study in terms of data collection, methods and analysis.

\section{Quantitative data collection}

The most useful tools for obtaining quantitative data in linguistics are the tests and questionnaires. The participant sample in empirical research is fundamental in guaranteeing a successful study. The sample is the group of people the researcher examines, the proper selection of which is crucial for drawing accurate conclusions. According to Dörnyei (2007), the sampling procedures are "probability sampling", typical for scientific research and "non-probability sampling", which are also common for qualitative research. Probability sampling includes a number of scientific procedures: random sampling, stratified random sampling, which combines randomization and categorization, systematic sampling refers to choosing every $\mathrm{n}^{\text {th }}$ member of a group, cluster sampling. Non- 
probability sampling includes three main strategies: quota and dimensional sampling, the former is a stratified one and the latter is a subset; snowball sampling is a chain nomination of members, convenience or opportunity sampling. The more scientific the investigation, the smaller the size.

\section{Questionnaire surveys}

The main and most frequently used tool and data collection method is the use of questionnaire surveys. They are easy to form and have a great potential for gathering a lot of information in a simple processible form. The essence of the research is attempting to find answers to questions in a systematic way and it is, consequently, logical that the questionnaire has turned to be the most popular research tool in quantitative research. The methodology is the following: 1) sampling the participants and 2) designing and managing the research instrument.

Questionnaires are defined by Brown as "any written instruments that present respondents with a series of questions or statements to which they are to react either by writing out their answers or selecting from among existing answers" (Brown, 2001, p. 6).

In general, questionnaires can produce three types of data about the respondent: -Factual questions are used to discover particular facts, usually demographic characteristics; - Behavioral questions are focused on finding personal history information; -Attitudinal questions cover people's thoughts, attitudes, opinions, beliefs and values. Questionnaires elicit information from the respondents without evaluating a performance based on certain criteria. In contrast, "production questionnaires", or the traditionally called "discourse completion tasks" (DCTs), used in interlanguage pragmatics research require the informants to produce some sort of authentic language data as a response to prompts. They are "structured language elicitation instruments" similar to language tests.

The main issue concerning questionnaires is their design. The way questionnaires are formulated is significant for the level of perception and sensitivity of the respondents which would affect the responses. The American psychologist Rensis Likert has discovered a genius way of overcoming the unexpected reactions to the wording of questionnaires by using "multi-item scales". That measurement of "language attitudes" is the foundational technique used most often in research today. According to Dörnyei (2007, p. 103) the "scales refer to a cluster of several differently worded items that focus on the same target" relying on different aspects of the item. In the end, the summation of the scores for the similar questions is averaged. Thus, multi-item scales "maximize the stable component that the items share and reduce the extraneous influences unique to the individual items" (Dörnyei, 2007, p. 104). 
Typically, the questionnaire is a structured data collection tool with items either asking for specific information or giving response options for the respondents to choose from. Most professional questionnaires are composed of "closed-ended" items in the form of multiple choice questions, allowing for the respondents to select an option. The most famous one is the "Likert scale", composed of a proposition and a range of possible answers measuring people's attitudes, opinions from "strongly agree" to "strongly disagree". Semantic differential scales are similar to Likert scale asking respondents to rate a target within the frame of bipolar adjectives. There are a lot of other closed-ended types, such as "Numerical rating scale", "True-False items", "Multiple-choice items", etc. Open-ended questionnaires, however, can be employed for some qualitative, exploratory studies since they require creative writing thus allowing more freedom in providing answers. The application of the method is exemplified in Ivanova and Gerova (2020, pp. 48-69; see also Ivanova, 2017).

The efficiency of questionnaires is their foremost strength for they make it possible for a large amount of data to be collected and processed for a very short time. They are also a quite flexible tool that could be used in various situations with different people, aimed at various topics. However, the method is considered as providing rather superficial data for its inconvenience in exploring matters thoroughly.

The experimental study is the most scientific quantitative research design because it manifests undeniable cause-effect relationships. The methodology is based on comparing data obtained from manipulated and controlled process with data - a result of similar process without any special control. The design is difficult to apply in educational contexts so the "quasi-experimental design" is a compromise with respect to the distribution of random assignment. It is similar to true experiments and the initial group-differences have to be decreased. The way to realize this is by avoiding the volunteer principle and minimizing pretest differences between the two groups. Two methods are used for realizing that objective:

- Matching participants in the treatment and control groups;

- Using analysis of covariance (AN COVA), which is the most frequently used statistical procedure (Lazaraton, 2005). This analysis evaluates the significance of the differences in the means of more than two groups (Dörnyei, 2007, p. 118).

\section{Qualitative data collection}

Qualitative data collection and analysis are not two separate phases but are often circular and overlap. It is difficult to decide whether a method refers to data collection or data analysis or a combined design - grounded theory and 
case studies are examples of this uncertainty. Qualitative procedures leading to the generation of a set of data are ethnography, one-to-one interviews, focus group interviews, introspection, case studies, diary studies and research journals (Dörnyei, 2007, p. 124). Qualitative data can be generated from various sources and it is always translated into a text. It is characterized by the huge amount obtainable and its heterogeneous nature. Hence, we must be careful in the selection of data: it has to be useful, realistic, following diverse strategies and "discovery-oriented" in the character of the inquiry.

In contrast to quantitative research, qualitative studies are concerned with describing and presenting individual aspects of the phenomenon in question. Thus, the main goal of sampling is focusing on people who can be potential suppliers of varied understanding of the issue explored, so that plenty of rich data would be collected. A qualitative research has to have a "purposeful" sampling with a plan that should be in accordance with the object of study. The process of sampling is preferable to remain open for a long time so that it could be alternated with the collection and analysis of initial accounts thus additional data is provided. The cyclical process of "moving back and forth" is referred to as "iteration" (Dörnyei, 2007, p. 126). The process should continue until we reach "saturation". Glaser and Strauss (1967) described this as "the point when additional data do not seem to develop the concepts any further but simply repeat what previous informants have already revealed. In other words, saturation is the point when the researcher becomes "empirically confident" (Dörnyei, 2007, p. 127). The most common strategies for achieving "saturation" are: homogeneous sampling, i.e. selecting participants with similar characteristics; typical sampling, i.e. participants sharing features typical with respect to the research focus; criterion sampling, maximum variation sampling, extreme/ deviant case sampling, critical case sampling, convenience sampling, etc.

\section{Ethnography}

The roots of ethnography could be traced back to anthropology and linguistic ethnography respectively draws on work in linguistic anthropology. Rampton et al. (2004) speak of "tying ethnography down and opening linguistics up" (Litosseliti, 2010, p. 139). Ethnographic research focuses on the description and analysis of the practices, values, behaviors, beliefs and languages of cultures. The notion of culture is intended to mean any "bounded units" (Harklau, 2005), such as organizations, communities and groups (qtd. in Dörnyei, 2007, p. 130). The approach is an excellent way of entering into "another culture" and "gaining insight into the life of organizations, institutions and communities" (Dörnyei, 2007, p. 133). Exploring unknown "territories" ethnography is successful in producing initial hypotheses which after the data collection phase are verified, developed and reviewed. In linguistic ethnography, regular patterns of action or 
talk of a group from a particular social context are identified and interpreted. "This is achieved through participant observation, field notes, ethnographic and open interviews, and often recordings/ transcripts" (Creese, qtd. in Litosseliti, 2010, p. 146).

According to Harklau(2005), a distinctive feature of classic ethnographic research is that it involves direct "participant observation" in a natural setting, and most studies that establish themselves as "ethnographic" include some degree of this method. Several features are emphasized as defining the ethnographic approach (Dörnyei, 2007, p. 131): 1) Focusing on participant meaning, i.e. the issue is looked at subjectively through the eyes of informants enabling the researcher to make a thorough study of a different culture.; 2) Prolonged engagement in the natural setting. The "in site" observation and interaction can help in identifying and explaining unexpected issues; 3) Emergent nature i.e. the research work progresses gradually after some field work has been done. The final product of the analysis is a "holistic cultural portrait of the group" that integrate the participants' and the researcher's views (Creswell, 2007, p. 72).

\section{Interviews}

It is the most frequently used method in qualitative research because it is an integral part of social life. The typical qualitative research interview has been described as a "conversation with a purpose" (Burgess, 1984, p. 102) and "the gold standard of qualitative research" (Silverman, 2000, p. 51). Its sharingknowledge essence makes it a "versatile instrument" in probing into an issue. The typical type of interview is one-to-one "professional conversation" (Kvale, 1996, p. 5) that has a structure and a purpose "to obtain descriptions of the life world of the interviewee with respect to interpreting the meaning of the described phenomena" (Dörnyei, 2007, p. 134).

Interviews take many forms, some are more structured, others - informal. The structured ones represent data collection in its most controlled form. They are similar to quantitative questionnaire, follow a guide and the questions are framed in advance so that variations are almost impossible. The context of the unstructured interview, known also as "ethnographic interview", is informal and the interviewer does not follow any preliminary guide. The interview is useful for in-depth exploration of a particular phenomenon or its development (Dörnyei, 2007, p. 136). A compromise is the semi-structured type that follows a guide but the interview can take unexpected turns and lead to interesting findings. This is the most frequently used type in linguistic research requiring the researcher to have general vision of the issue or the sphere explored. The key principle in interview methodology is the neutrality of the interviewer, not affected by personal bias. 


\section{Focus group interviews}

They are often considered a subtype of interviews and are useful in many different spheres relevant to qualitative research. As its name suggests focus groups are always characterized with multiple respondents engaged in a collective talk centered around a limited number of issues. It involves "within group" interaction that results in large amount of top-quality data. The collective brainstorming elicits discussions and may "spiral off" in various directions while the researcher acts more as a facilitator than as an interviewer. Heterogeneous groups are valuable with regard to obtaining diverse data and examining different perspectives of the issue explored.

\section{Introspective methods}

As a result of psychological research at the end of $19^{\text {th }}$ century, the focus of which was the conscious mind of a person or more exactly the mental processes that go through the mind, introspection appeared as a term, including the various ways of eliciting information about thought processes. Nunan (1992), defines introspection as "the process of observing and reflecting on one's thoughts, feelings, motives, reasoning processes, and mental states with a view to determining the ways in which these processes and states determine our behavior" (Heigham \& Croker, 2009, p. 220). Gass and Mackey (2000) point out that "the assumption underlying introspection is that it is possible to observe internal processes", that is, what is going on in one's consciousness, in much the same way as one can observe external real-world events (Dörnyei, 2007, p. 147). Introspective methods are seen to be relevant to applied linguistics because language is what represents consciousness on the surface. Kormos (1998) emphasizes their importance in second language learning. Introspective methods include two techniques: "think aloud" and "retrospective reports". The proponent of introspective methods in psychology, Ericsson (2002), analyzes the relation between mental processes and verbal reports. In the "think aloud" technique respondents are asked to verbalize their stream of thoughts while performing a task. In the "retrospective interview" the respondents express their thoughts after the completion of the task and the validity of data depends on the time interval between the two actions. For that reason, Mackey and Gass (2005) recommend the "retrospective interview" or the "stimulated recall". There is some kind of stimulus used for the retrieval process that can help activate respondents' short/ long-term memory. (Dörnyei, 2007, p. 149). Verbal reports are considered valuable storage of cognitive processes which are further analyzed depending on the kind of issue that is investigated. The advantages of the introspective methods in relation to linguistics is that it gives access to language production resulting from mental processes. It could be used in combination with other methods, increasing the amount of data and its reliability. 


\section{Case Studies}

The case study is a type of research design and analysis which is generally characterized as the most widely used approach in qualitative research in linguistics and education. The notion of "case study" could be defined as the study of the "particularity and complexity of a single case" (Stake, 1995, p. xi). A case is predominantly a person and could be anything representing a singular unit with apparent boundaries or a "bounded unit". The focus of case studies is to provide an in-depth description and analysis of a case (or cases) using multiple data sources, such as interviews, observations, diaries, verbal reports, etc. The task of the researcher is to explore and identify the boundaries of the case regarding the focus and interest of the researcher. Each study aims at exploring one singular case, thus, it can be divided into multiple levels of research which justifies the use of different methods, so as to fully enhance our comprehension of the issue studied. Stake $(1995,2005)$ points out three types of case study: 1) the intrinsic case study is undertaken to explore the inner nature of a particular case for its own sake; 2) instrumental case study provides insight into a wider matter and the actual case is not of primary concern. According to Merriam (1998) the intrinsic case study entails a descriptive approach with a focus on particularity whereas the instrumental one also involves evaluation and interpretation. It supports, proves or challenges theoretical statements (Heigham \& Croker, 2009, p. 70); 3) the multiple or collective case study focuses on several cases to study the general conditions of one issue which makes it similar to instrumental case study. Thus, we can gain knowledge about a bigger collection of cases. The case study researcher Yin (2003) offers still other three types based on the objectives of the study: exploratory, descriptive and explanatory (Heigham \& Croker, 2009, p. 70). The effective case study researcher must be a careful observer for s/he is the "filter", deciding which data is valuable to serve the aim of the study. We run the risk of distorting the data due to our preconceived opinion and a research diary could be of great help in maintaining a critical attitude on the data. An application of this research design can be observed in the analysis provided by Radostina Iglikova and Olga Usataya on the translation of movie titles in English into Russian (Iglikova \& Usataya, 2019; see also Seizova-Nankova, 2016; Iglikova, 2017).

\section{Research journals}

Keeping track of one's thoughts, ideas, suppositions during the research process is important for every researcher. Hence, the journal or diary are the perfect assistants and a valuable source of data. Dörnyei views the journal as "the vehicle to transform private knowledge, by reflection and analysis, into potential public knowledge" (2007, p. 160). Research journals play a significant role in maintaining validity and reliability in qualitative research for they reveal each step in the project and prove the ideas and theories. It takes some discipline 
to keep a journal but it repays the efforts for it can become a "platform for conceptualizing, noticing, articulating, or testing out new hypotheses or ideas" (Schmidt \& Frota, 1986). Altrichter and Holly (2005) highlight that an important aspect of the research journal as a data source is to include "descriptive sequences" such as accounts of activities and events, or reconstructions of dialogues. In such accounts, the authors underline, "details are more important than summaries and we need to focus on the particular rather than the general" (Dörnyei, 2007, p. 161).

\section{Qualitative data analysis}

The analysis of qualitative data is seen as characterized by great diversity and there are several principles that are considered essential for the mastery of the process. The first point to stress is "the language-based nature of the analysis", i.e. the data is transformed into words. The second principle defines the "iterative" and "non-linear" nature of the analysis, i.e. the researcher moves back and forth between the data collection, analysis and interpretation depending on the results. Crucial for the data analysis is "saturation" and the process should stop when there are not any new ideas, themes and topics emerging. Another central point in terms of qualitative analytical approach is its reliance on subjective intuition than following formalized procedures. The analysis has to be flexible, "artful", allowing for new ideas to emerge and flow freely without the limitations of traditional procedures. However, opponents of that approach claim that only systematic procedures help in achieving valid conclusions. Thus, it becomes a challenge for the researcher to conform to "disciplined artfulness" when applying the methods valuable for producing new theories. The last essential principle underlying the qualitative data analysis is the use of "general analytical moves "or it is often termed as "qualitative content analysis" as opposed to the use of specific methodology.

\section{Qualitative content analysis}

The roots of content analysis can be found in a quantitative analytical method of examining written texts that requires the counting of words, phrases, or grammatical structures that are grouped under specific categories. However, the categories in the content analysis are not predetermined but are obtained by inductive reasoning from the collected data (Dörnyei, 2007, p. 245). Qualitative content analysis is referred to as "latent level analysis" because it is a deeper level of analysis and interpretation of the underlying meaning of the data. There are four phases of content analysis: 1) transcribing the data, 2) pre-coding and coding - pre-coding reflections shape our ideas about the data and would lead to forming the themes of the project. Dörnyei argues that "all the qualitative coding techniques are aimed at reducing or simplifying the data while highlighting 
special features of certain data segments in order to link them to broader topics or concepts" (2007, p. 250). Coding should be accompanied by some analytical tools that can help to develop the final themes, the most useful of which is writing memos. These analytic memos are the seeds that will give rise to the main conclusions. The process of interpreting the data and drawing conclusions incorporate taking account of everything we have, evaluating the collected patterns, concepts and ideas, and selecting the final themes which to develop in detail (see for example Mazahir, Yaseen \& Siddiqui, 2019).

\section{Grounded Theory}

For many scholars "grounded theory" is "a common methodological framework for qualitative research in general" (Arksey \& Knight, 1999, see also Chun Tie, Birks \& Francis, 2019; Thurlow, 2020) since it provided theoretically-based and complex methodology trying to undermine the dominance of quantitative research. Grounded theory has grown into the "mainstream" and many of its theoretical aspects have turned into "core issues" in qualitative research. The principal aim of the method is to create a new theory framed on the basis of empirical data or as Creswell points out "the intent of a grounded theory study is to move beyond description and to generate or discover a theory, an abstract analytical schema of a process (2007, p. 59). The question of the validity of a theory is debatable and Fassinger (2005) claims that if the result of the research is "a coherent, contextualized explanation (rather than merely a contextual description) of an issue, possibly also outlining a (tentative) model or framework" (Dörnyei, 2007, p. 260), then it could be accepted as a theoretical product.

A characteristic aspect of the grounded theory is the differentiation between the different phases of coding the data. The system of coding consists of three distinct levels. The first one is "open coding" in which the data is split into pieces that are categorized. The emphasis is on provoking new ideas rather than on description and interpretation. In the second level, called "axial coding", interconnections between categories are established resulting in more embracing concepts that include several items. Creswell recommends at this level that we return and reanalyze the data in order to describe the links between the categories (Dörnyei, 2007, p. 261). That would provide the basis for the third level of "selective coding" aimed at selecting a "core category" which will be the focus of analysis henceforth and the central theme of the theory. A coherent abstract "core" that encompasses the other concepts ensures an in-depth "inductive" analysis of a phenomenon which moves on to a theory grounded on that specific mode of analysis. An example of qualitative research in which data received from focus-group interviews were analysed by means of grounded theory could be found in Ivanova's chapter on teacher-trainees' preferences for explicit evaluation (Ivanova, 2020, pp. 197-222). 


\section{Discourse-Analytic Approaches}

The term "discourse" is defined in a number of ways: "verbal interchange of ideas", "formal and orderly and usually extended expression of thought on a subject", "connected speech or writing", "a linguistic unit larger than a sentence" (Merriam-Webster, n.d.). For Fairclough (1995) "discourse" is the use of language seen as a form of social practice, a social activity and mutual influence, people who interact in real social situations [as well as] the social creation of reality, a kind of knowledge" $(1995, \mathrm{p} .18)$. It is also viewed as a text, functioning in a specific sphere of social life (Todorova, 2015). In the words of Jaworski and Coupland (1999, p. 49) discourse is a "form of collaborative social action" in which language users "jointly collaborate in the production of meanings and inferences" as they communicate.

Discourse-analytic approaches reassess and examine the use of language in spoken and written communication. Chomsky, who is the father of modern linguistics, postulated that the aim of linguistics is to study "linguistic competence" i.e. the unconscious knowledge of grammar or the rules governing language use. Del Hymes (1972) introduced the notion of "communicative competence" as opposed to "linguistic competence" involving not only mastery of the language code but also knowledge of appropriate language use. That notion is important in discussing spoken and written discourse, taking account of the social and cultural setting.

There are two main fields of discourse-analytical methodology: microanalytical approaches, dealing with "the finer detail of linguistic interactions in transcripts", and macroanalytical approaches, "which consider how broader social processes work through language" (Heller, 2001, qtd. in Litosseliti, 2010, p.119). The following four approaches are the most significant in the field and the most salient in presenting the contrast between the two trends: microanalytical approaches (Conversation analysis) and macroanalytical approaches (Critical discourse analysis) as well as that which combines (Dscourse analysis) or challenges the aspects of both (Feminist Post-structuralist Discourse Analysis).

\section{Conversation Analysis (CA)}

$\mathrm{CA}$ is at the other extreme of Chomsky's view related to the focus of linguistic study. Spoken interactions dominate our world and the exploration of "talk-ininteraction" provides abundant data of the underlying rules of language. The sociolinguist Sacks (1992) was the first to explore utterances as "social actions which speakers use to get things done (or to avoid getting things done) in the course of a conversation with others" (Litosseliti, 2010, p. 121). CA postulates that social interactions are the creators of reality and arena for human actions. Its goal is to discover and describe interactional architecture. A guiding assumption of research in $\mathrm{CA}$ is that the natural setting of language is "co- 
present interaction" and that its structure is basically adapted to that setting. While talking we perform actions which instigate subsequent ones and thus form a connected series of actions. Language in action has a social organization and it is this "sequence" in interaction that is the focus of investigation. The following features characterize the essence of research in CA: 1) Orderliness in talk-in-interaction, known as the "speech-exchange system", which includes patterns of conversational openings and closings, turn taking, sequence of connected utterances ("adjacency pairs"), feedback and conversational "repair". The interest is, as Paltridge explains, in "fine-tuned analysis" of structure and coherence of interactions (Paltridge, 2006, p. 110; see also Litosseliti, 2010, p. $121)$; 2) CA is a data-centered approach, studying only naturally occurring language and the actions people perform with their speech in real-life situations. The CA transcriptions are valuable resources for analysis of data because they record details (increased pitch, pauses, sequence of utterances) significant for the progress of interaction and for a detailed analysis of meaning production; 3 ) neutrality and objectivity: prior presuppositions to the data, speculations about participants' identities, social settings and norms are considered irrelevant.

CA can be employed as a valuable tool in cross disciplinary studies by using methods of microanalysis, operating from the bottom to the top. It attributes special value to the linguistic data and considers itself a caution against the "relativisation and perspectivisation of cultural analysis" (Schegloff, 1997, p. 183). An example of the application of this method of analysis could be seen in Peneva (2016; see also Peneva, 2017).

\section{Discourse Analysis (DA)}

Discourse analysis for Brown and Yule is an investigation of what language is used for, i.e. its functions. They identify the two main functions of language: "transactional" or expressing "content" and "interactional", displaying social relations and attitudes (Brown \& Yule, 1983, p. 1). The term discourse analysis was introduced by Zelig Harris as a way of analyzing connected speech or writing. He was interested in language "beyond the level of sentence", especially in the ways language features are distributed in texts. Another concern of Harris is "the relationship between linguistic and non-linguistic behavior" (Paltridge, 2006, p. 2) which refers to the analysis of language in use, or how the interpretation of language depends on the situation in which it is used. There are various ways of using language in particular situations and the linguistic features characterizing them as well as the realizations of meaning in language is the primary focus of DA.

DA explores the relationship between language and the context (social or cultural) it is employed in, considering both spoken and written language. DA is interested more in the meaning of utterances than in the utterances themselves, 
which is also an aspect of Pragmatics. DA examines stretches of text and how they are organized. Mitchell (1957) was the first to study the discourse structure of texts, i.e. the particular ways of ordering what we say or the overall textual organization. He introduced the term "stages "in DA to mean the definite phases we go through in particular spoken or written situations, languages and cultures" (Paltridge, 2006, p. 4). The social and cultural settings in which the speaking or writing occurs is a key issue in DA in comprehending the language choice. It is closely related to Hyme's notions of "ethnography of communication" and "communicative competence" which examine different aspects of speech events (interactions) and their impact on language in different cultural settings.

There are different views on what DA is. Cazden (1998) summarizes two main views: those which examine the natural flow of language and those which focus on different ways of talking and understanding. Fairclough (2003, p.36) contrasts "textually-oriented DA" with language in action. He and other researchers do not view these perspectives as contradictory. Therefore, DA is a view of language at textual level and also an aspect of language in use, i.e. how through language people achieve communicative goals, perform acts, present themselves to others, communicate with other cultures and groups, etc. DA studies texts as communicative units, embodied in social and cultural practices or as Paltridge puts it "discourse is shaped by the world and shaping the world" (2006, p. 9). DA is concerned with the effect the use of language has on social identities and relations and how these influence the use of language. DA takes us into realizing the "bigger picture" of language description (Riggenbach, 1999). It takes us into the social and cultural context of discourse to help us understand the language choices (Partridge, 2006, p. 20). Discourse-analytic approaches depend on the different perspectives taken and the issues examined in the research process (see also Johnstone, 2008; Tannen, Hamilton \& Schiffrin, 2018).

\section{Discourse and Grammar}

Research has provided evidence of patterns of language examined from a discourse perspective and proved that linguistic items exhibit different functions. A focus of Discourse grammar is on larger units of text than isolated words, phrases or sentences, i.e. it has moved beyond sentence level. A key point is the role the language items have in the entire discourse and how they combine to link meanings in a text, i.e. to make a cohesive and coherent unit. The focus of Discourse grammarare such language aspects as: reference, cohesion, collocation, conjunction, substitution and ellipsis, theme-rheme and thematic progression, which contribute to the texture of a text (Paltridge, 2006, p. 130). Hilles (2005) studies discourse and grammar from a contextual perspective, paying special attention to the socio-interactional or contextual factors that may affect and determine the use of a particular linguistic item in the discourse (Paltridge, 2006, p. 129). Discourse-based grammar relates form, function and context, and 
places appropriateness and use as the central issue of its descriptions (Hughes \& McCarthy, 1998). Similar view is presented by Larsen-Freeman (2003) claiming that form, meaning and use are indispensable for establishing the base of all descriptions. Discourse-based grammar recognizes the importance of language choices, fosters cognition of interpersonal factors and gives insights into aspects of grammar that could not be reasonably explained.

\section{Critical Discourse Analysis (CDA)}

CDA is a new democratic approach to the study of texts that emerged at the end of $20^{\text {th }}$ century and can be seen as "a problem-oriented interdisciplinary research programme, subsuming a variety of approaches" (Wodak, 2013, p. xx). All research methods share the same idea and interest in the "semiotic dimensions of power, injustice and political-economic, social or cultural change in our globalized and globalising world and societies (Wodak, 2013, p. xix). CDA is concerned with the study of language and its relation to power and ideology in the social, institutional and political contexts in which it occurs. CDA research considers the use of discourse in relation to issues such as: gender, ethnicity, cultural differences, ideology, identity, politics, inequality, racism and how these are organized and reflected in texts. According to Van Dijk, CDA aims to explore, reveal and challenge some hidden ideologies, positions, perspectives and values that underlie texts (2001, p. 352). He argues that CDA defines the influence of the social contexts on language diversity and the discourse social variables such as, gender, race, age, etc. Awareness of the inherent properties of communicative situations affects language. Thus, the study of discourse tries to strike a balance between society, culture, situation and language. Wodak and Mayer (2009, p. 7) stress the need for interdisciplinary work in relation to $\mathrm{CDA}$ in order to grasp how language works in constituting and transferring knowledge, "in organizing social institutions or in exercising power". For Van Leeuwen, CDA views discourse as a "conductor of power and control" and "an institution for a social creation of reality" (1993, p. 793). It focuses mainly on the analysis and critique of unfair social practices. A critical analysis may include examining the text thoroughly, and then moving to analysis and interpretation. Adding systematicity to analysis, Todorova claims that CDA "incorporates three levels of analysis: the text, the discursive practices that create and interpret it and the larger social context relevant to it" (Todorova, 2015, p. 189).

There is no consensus concerning the definition of CDA. However, Fairclough and Wodak give a brief summary of the main principles that CDA is based upon: 1) discourse is constitutive and reflective of social and political issues; 2) power relations are brought into play and performed through discourse; 3) discourse is part of social relations, establishing and maintaining them. It also fosters social and gendered stereotypes and inequalities (2003, p. 4) ideologies are formed and displayed within the frames of discourse (Paltridge, 2006, p. 179). 
Though CDA has a diverse nature it is mainly used in institutional discourse and the language of media. An illustrative example of the application of CDA can be seen in Cheshmedzhieva-Stoycheva (2020b; see also CheshmedzhievaStoycheva, 2017; Cheshmedzhieva-Stoycheva, 2016).

\section{Feminist Post-Structuralist Discourse Analysis (FPDA)}

FPDA has its origin in feminist post-structuralism and is developed as a method of analysis by Baxter. It can be defined as: an approach to analyzing intertextualised discourses in spoken interaction and other types of text. It draws upon the poststructuralist principles of complexity, plurality, ambiguity, connection, recognition, diversity, textual playfulness, functionality and transformation. The feminist perspective on poststructuralist discourse analysis considers gender differentiation to be a dominant discourse among competing discourses when analysing all types of text (Baxter, 2008, p. 245, qtd. in Litosseliti, 2010, p. 130). The poststructuralist part of FPDA considers discourses as forms of organizing power relations between speakers, and the analysis examines the ways discourses work intertextually positioning participants as powerful and powerless, frequently changing their positions. The feminist part of FPDA emphasizes the social category of gender with regard to the ways in which power relations are created through spoken interaction. Hence, the principal function of FPDA to foreground discourses of gender difference as they are performed within specific contexts. The following are the key principles FPDA is based upon: 1) Continuous reflexivity, i.e. the need to be critical about values and suppositions of discourse analysis; 2) FPDA does not have an "emancipatory agenda" for women but a "transformative quest" (Baxter, 2003). This means that it aims to represent the complex nature of female experience and women's voices that have been "silenced" or marginalized since FPDA acknowledges that these have been absent in many cultures. Thus, FPDA is in favour of small scale, localized transformations that challenge dominant discourses; 3) Diversity of speakers' identities: for FPDA, identities are formed by means of multiple variables, denoting power such as race, ethnicity, age and gender, which is the dominant among these. Due to context, some of the variables are more notable than others in creating identities through interaction; 4) FPDA questions the tendency of polarized subjects, the powerful and powerless, but posits that gender identities are complex and unstable in their positions of power. 5) An interaction between a fine-grained analysis and macroanalysis, FPDA examines the linguistic data regarding turn-taking, sentence structure, verb tense, lexical choice, coherence and cohesion, aspects which help analysts to identify the exact moments of shifts between states of powerfulness and powerlessness, along with explanations of shifts of power relations between participants within particular contexts. An application of this method of analysis can be seen in 
Cheshmedzhieva-Stoycheva's (2020a) research on the development of the image of Ilhan Omar in American media.

\section{Conclusion}

In summary, the paper aims at providing a brief theoretical overview of the principal research paradigms, key issues, procedures, data collection and methods of analysis applied to the study of language. Research involves collecting data, analyzing it and making inferences from analysis. General description of qualitative, quantitative and mixed-method research is followed by more detailed account of the three approaches. Specific for quantitative research is going beyond the particular in the pursuit for generalizing the facts. In short, quantitative research has strengths that are indisputable - it is systematic, controlled, involving exact measurements resulting in reliable and generalized results. The foremost feature of qualitative research is its emergent nature, i.e. no aspect of the research design is predicted, the study is flexible and open to new details that may emerge during the process. Mixed methods research can be specified as a combination of quantitative and qualitative methods in a single project. The two methods can supplement and inform each other, thus providing higher quality results and better comprehension of a phenomenon. Quality criteria in quantitative research are validity and reliability. Setting quality standards in qualitative research is problematic because the study is subjective, discursive and context-specific. Two taxonomies of quality criteria in qualitative research are proposed. Lincoln and Guba suggest four components that "trustworthiness" is composed of: credibility, transferability, dependability and confirmability. Maxwell proposed five components of validity: descriptive, interpretative, theoretical, evaluative validity and generalizability. The logical way to do research includes generating the research questions, choosing the design, then the method and the instruments allowing for the questions to be answered. Realizing the possible outcomes of the investigation we can narrow down the scope of the research purpose to "research hypotheses". The main and most frequently used tool and data collection method is the use of questionnaire surveys. The efficiency of questionnaires is their foremost strength for they make it possible for a large amount of data to be collected and processed for a very short time. Qualitative data collection and analysis are not two separate phases but are often circular and overlap. Various data collection methods are considered: ethnography, interviews, focus groups interviews, introspection, case studies and research journals. A central aspect in qualitative data analysis is the reliance on objective intuition than following formalized procedures. Qualitative content analysis is a deeper level of analysis and interpretation of the underlying meaning of the data. Grounded theory has grown into the "mainstream" and many of its theoretical aspects have turned into "core issues" in qualitative research. The principal aim of the method is 
to create a new theory framed on the basis of empirical data. There are two main fields of discourse-analytical methodology: microanalytical, dealing with "the finer detail" of linguistic interactions and macroanalytical, which consider how social processes work through language. The most significant in the field are: Conversation analysis, Discourse analysis, Critical discourse analysis and Feminist Post-Structuralist Discourse analysis. The different discourse-analytic approaches, positioned within different paradigms, are likely to produce varying accounts of the same data thus producing richer and complex insights within linguistic research.

\section{References:}

Arksey, H., \& Knight, P. (1999). Interviewing for social scientists. London: Sage.

Bachman, L. F. (2004). Linking observations to interpretations and uses in TESOL Research. TESOL Quarterly, 38(4), 723-728.

Baxter, J. (2010). Discourse-analytic approaches to text and talk. In L. Litosseliti (Ed.) Research Methods in Linguistics (pp. 117-137). London: Continuum.

Bazeley, P. (2003). Computerized data analysis for mixed methods research. In A. Tashakkori \& C. Teddlie (Eds.), Handbook of Mixed Methods in Social and Behavioral Research (pp. 431-469). Thousand Oaks, Calif.: Sage.

Brannen, J. (2005). Mixed methods research: A Discussion Paper. Southampton: ESRC National Centre for Research Methods.

Brewer, J., \& Hunter, A. (1989). Multimethod research: A synthesis of styles. Newbury Park, Calif:: Sage.

Brown, G., \& Yule, G. (1983). Discourse analysis. Cambridge: Cambridge University Press.

Brown, J. D. (2001). Using surveys in language programs. Cambridge: Cambridge University Press.

Chalhoub-Deville, M. (2006). Drawing the line: The generalizability and limitations of research in applied linguistics. In M. Chalhoub-Deville, C. A. Chapelle \& P. Duff (Eds.). Inference and Generalizability in Applied Linguistics: Multiple Perspectives (pp. 1-9). Amsterdam: John Benjamins.

Cheshmedzhieva-Stoycheva, D. (2016). Idle, stricken, or retired: Challenges in understanding media discourse on nuclear power. Studies in Linguistics, Culture, and FLT, 1, 165-177. doi: 10.46687/SILC.2016.v01.015.

Cheshmedzhieva-Stoycheva, D. (2017). Stereotypes and prejudices at the border: an attempt at raising intercultural awareness through examples from the Bulgarian and the British media. Studies in Linguistics, Culture, and FLT, 2, 184-197. doi: 10.46687/SILC.2017.v02.015.

Cheshmedzhieva-Stoycheva D. S. (2020a). Media discourse on women in politics: The first Muslim woman of colour in US Congress. Media Linguistics, 7(4), 490-500. https://doi.org/10.21638/spbu22.2020.408. 
Cheshmedzhieva-Stoycheva, D. (2020b). Aspects of media presentations on Russians: yet another spy story in the media. Studies in Linguistics, Culture, and FLT, 8(2), 19-51. doi: 10.46687/SILC.2020.v08i02.002.

Chun Tie, Y., Birks, M., \& Francis, K. (2019). Grounded theory research: A design framework for novice researchers. SAGE Open Medicine, 7, 205031211882292. doi:10.1177/2050312118822927.

Creswell, J. (2007). Qualitative inquiry and research design: Choosing among five approaches. Thousand Oaks, CA: Sage.

Denzin, N. K. (1978). The research act: A theoretical introduction to sociological methods (2nd ed.). New York, NY: McGraw Hill.

Denzin, N. K., \& Lincoln, Y. S. (2005). Introduction: The discipline and practice of qualitative research. In N. K. Denzin \& Y. S. Lincoln (Eds.). The Sage Handbook of Qualitative Research (3rd ed.). Thousand Oaks, Calif.: Sage.

Denzin, N. K., \& Lincoln, Y. S. (Eds.). (2011). The Sage handbook of qualitative research. Thousand Oaks: Sage.

Dörnyei, Z. (2007). Research methods in applied linguistics. Quantitative, qualitative and mixed methodologies. Oxford: Oxford University Press.

Duff, P. (2006). Beyond generalizability: contextualization, complexity, and credibility in applied linguistics research. In M. Chalhoub-Deville, C. A. Chapelle \& P. Duff (Eds.). Inference and Generalizability in Applied Linguistics: Multiple Perspectives (pp. 65-95). Amsterdam: John Benjamins.

Ericsson, K. A. (2002). Towards a procedure for eliciting verbal expression of non-verbal experience without reactivity: interpreting the verbal overshadowing effect within the theoretical framework for protocol analysis. Applied Cognitive Psychology, 16, 981-987.

Fairclough, N. (1995). Critical discourse analysis: The critical study of language. London: Longman.

Fairclough, N., \& Wodak, R. (1997). Discourse as social interaction. London: Sage.

Johnstone, B. (2008). Discourse analysis (2nd ed.). Malden, MA: Blackwell Publishing.

Hatch, E. M., \& Lazaraton, A. (1991). The Research manual: Design and statistics for applied linguistics. New York: Newbury House.

Heigham, J., \& Croker, R. (2009). Qualitative research in applied linguistics. A practical introduction. London: Palgrave Macmillan.

Hughes, R., \& McCarthy, M. (1998). From sentence to discourse: Discourse grammar and English language teaching. TESOL Quarterly, 32, 263-287.

Iglikova, R. (2017). Headline patterns in viral web content - English-Bulgarian comparative case study. Studies in Linguistics, Culture, and FLT, 2, 71-84. doi: 10.46687/SILC.2017.v02.006.

Iglikova, R., \& Usataya, O. (2019). Strategies and techniques in the translation of movie titles (an English-Russian comparative case study). Studies in Linguistics, Culture, and FLT, 7, 59-70. doi: 10.46687/SILC.2019.v07.005. 
Ivanova, I. (2017). Teachers' awareness of the benefits of continuing professional development. Studies in Linguistics, Culture, and FLT, 2, 205-218. doi: 10.46687/ SILC.2017.v02.017.

Ivanova, I. (2020). Making sense of research: university students' difficulties in writing academic summaries. Studies in Linguistics, Culture, and FLT, 8(1), 16-34. doi:10.46687/SILC.2020.v08i01.002.

Ivanova, I., \& Gerova, G. (2020). Bulgarian students' perceptions of issues and challenges in preparing for the English language maturity exam. Studies in Linguistics, Culture, and FLT, 8(3), 48-68. doi: 10.46687/SILC.2020.v08i03.004.

Jaworski, A., \& Coupland, N. (1999) Introduction: Perspectives on discourse analysis. London: Routledge.

Kormos, J. (1998). Verbal reports in L2 speech production research. TESOL Quarterly, 32, 353-358.

Larsen-Freeman, D. (2003). Teaching language: From grammar to grammaring. Boston, MA: Thomson Heinle.

Lazaraton, A. (2005). Quantitative research methods. In E. Hinkel (Ed.). Handbook of Research in Second Language Teaching and Learning (pp. 209-224). Mahwah, N.J.: Lawrence Erlbaum.

Litosseliti, L. (Ed.). (2010). Research methods in linguistics. London: Continuum.

Merriam, S. (1998). Qualitative research and case study applications in education. (2nd ed.). San Francisco, CA: Jossey-Bass.

Middleton, F. (2019). Types of reliability and how to measure them. Retrieved March 25, 2021, from https://www.scribbr.com/author/fionamiddleton/.

Miles, M., \& Huberman, A. (1994). Qualitative data analysis: An expanded sourcebook. Thousand Oaks: Sage.

Aronoff, M., \& Clark, R. (Eds.). (2021). Oxford Research Encyclopedia of linguistics. Retrieved March 24, 2021, from https://oxfordre.com/linguistics.

Mazahir, I., Yaseen, S., \& Siddiqui, M. (2019). International comparison of media coverage on the Fukushima crisis: A COMPARATIVE content analysis of news media coverage in several countries. Media Education (Mediaobrazovanie), 59(4), 557-573. doi:10.13187/me.2019.4.557.

Morse, J. M., \& Richards, L. (2002). Readme first for a user's guide to qualitative research. Thousand Oaks, Calif.: Sage.

Peneva, D. (2016). Challenges in teaching remedial apologies to learners of English as a foreign language. Studies in Linguistics, Culture, and FLT, 1, 117-124. doi: 10.46687/ SILC.2016.v01.011.

Peneva, D. (2017). The use of commissive speech acts in English and Bulgarian and their politeness implication. Studies in Linguistics, Culture, and FLT, 2, 227-239. doi: 10.46687/SILC.2017.v02.019.

Podesva, R., \& Sharma, D. (2013). Research methods in linguistics. Cambridge University Press.

Richards, L. (2005). Handling qualitative data: A practical guide. London: Sage Publications. 
Rossman, G. B., \& Wilson, B. L. (1985). Numbers and words: combining quantitative and qualitative methods in a single large-scale evaluation study. Evaluation Review, 9(5), 627-643.

Seizova-Nankova, T. (2016). Valency constructions at work: a case study. Studies in Linguistics, Culture, and FLT, 1, 21-41. doi: 10.46687/SILC.2016.v01.003.

Silverman, D. (2000), 'Analyzing talk and text', in N. K. Denzin and Y. S. Lincoln (eds), Handbook of Qualitative Research (2nd ed.). London: Sage.

Silverman, D. (2005). Doing qualitative research (2nd ed.). London: Sage.

Schegloff, E. A. (1997). Whose text? Whose context? Discourse \& Society, 8, 165-187. Sage Publications, Inc.

Schmidt, R., \&. Frota, S. F. N. (1986). Developing basic conversational ability in a second language: A case study of an adult learner of Portuguese. In R. Day (Ed.), Talking to learn: Conversation in second language acquisition (pp. 237-369). Rowley, MA: Newbury House.

Stake, R. E. (1995). The art of case study research. Thousand Oaks, Calif.: Sage.

Tannen, D., Hamilton, H. E., \& Schiffrin, D. (2018). The handbook of discourse analysis. Chichester: Wiley Blackwell.

Tashakkori, A., \& Teddlie, C. (1998). Mixed methodology: Combining qualitative and quantitative approaches. Thousand Oaks, Calif.: Sage.

Teddlie, C., \& Tashakkori, A. (2003). Major issues and controversies in the use of mixed methods in the social and behavioral sciences. In A. Tashakkori \& C. Teddlie (Eds.). Handbook of Mixed Methods in Social and Behavioral Research (pp. 13-50). Thousand Oaks, Calif.: Sage.

Thurlow, L. (2020). Grounded theory and the $\mathrm{PhD}$ - notes for novice researchers. Journal of Humanities and Applied Social Sciences, 2(4), 257-270. doi:10.1108/ jhass-05-2020-0079.

Todorova, R. (2015). Insights in text linguistics. From theory to practice. Shumen: Konstantin Preslavski University Press.

van Dijk, T. (2001). Multi-disciplinary CDA: A plea for diversity. In R. Wodak \& M. Meyer (Eds). Methods of Critical Discourse Analysis (pp. 1-13). London and Thousand Oaks, CA: Sage.

Hassen, R. (2015). Discourse as medium of knowledge: transmission of knowledge by transmission of discourse people live. Journal of Education and Practice, 6(31), 119-128.

Wodak, R. (2013). Critical discourse analysis. London, UK: Sage

Wodak, R., \& Meyer, M. (Eds). (2009). Methods of critical discourse analysis (2nd ed.). London: Sage.

Wooffitt, R. (2005). Conversation analysis and discourse analysis: A comparative and critical introduction. Thousand Oaks: Sage

Merriam-Webster. (n.d.). Discourse. In Merriam-Webster.com dictionary. Retrieved March 24, 2021, from https://www.merriam-webster.com/dictionary/discourse. 